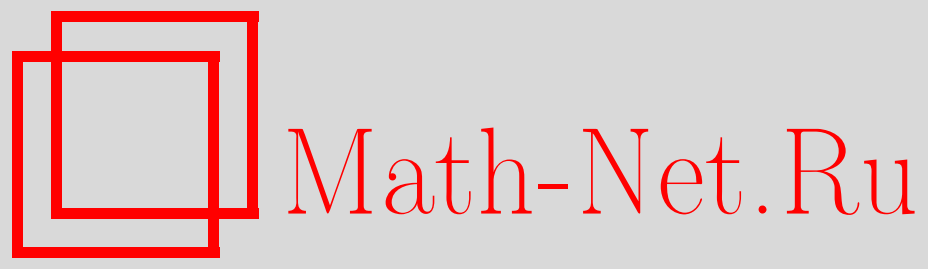

M. G. Benli, Р. И. Григорчук, Т. В. Нагнибеда, Универсальные группы промежуточного роста и их инвариантные случайные подгруппы, Функи. анализ и его прил., 2015, том 49, выпуск 3, 1-21

DOI: https://doi.org/10.4213/faa3205

Использование Общероссийского математического портала MathNet.Ru подразумевает, что вы прочитали и согласны с пользовательским соглашением http://www . mathnet.ru/rus/agreement

Параметры загрузки:

IP : 3.89 .185 .249

26 апреля 2023 г., 11:24:44

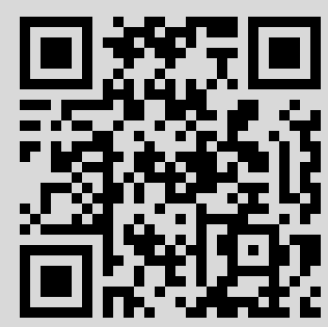


Функционалъный анализ и его приложения

2015, т. 49, вып. 3, с. 1-21

УДК 512.54

\title{
Универсальные группы промежуточного роста и их инвариантные случайные подгруппы*
}

\author{
(c) 2015. М. Г. БЕНЛИ, Р. И. ГРИГОРчУК, Т. В. НАГНИБЕДА
}

Анатолию Моисеевичу Вершику по случаю его 80-летия с восхищением и глубоким уважением

В работе приведены примеры групп промежуточного роста с $2^{\aleph_{0}}$ эргодическими непрерывными инвариантными случайными подгруппами. Эти примеры - универсальные группы, ассоциированные с некоторым семейством групп промежуточного роста.

\section{§1. Введение}

Цель данной работы - продемонстрировать существование групп промежуточного роста с континуумом эргодических непрерывных инвариантных случайных подгрупп.

Инвариантная случайная подгруппа (сокращенно ИС-подгруппа) - удобный термин для инвариантной относительно действия группы сопряжениями вероятностной меры на пространстве подгрупп локально компактной группы. В случае счетной группы $G$ (а здесь только такие и будут рассматриваться) пространство $S(G)$ подгрупп группы $G$ снабжается топологией, индуцированной топологией Тихонова на прямом произведении $\{0,1\}^{G}$ при отождествлении подгруппы $H \leqslant G$ с ее характеристической функцией $\chi_{H}\left(\chi_{H}(g)=1\right.$ при $g \in H$ и 0 в противном случае).

Дельта-масса, соответствующая нормальной подгруппе, является тривиальным примером ИС-подгруппы, равно как и среднее по орбите дельта-масс, соответствующих подгруппам из некоторого конечного класса сопряженности. Поэтому мы будем интересоваться непрерывными инвариантными вероятностными мерами на $S(G)$. Ясно, что такие меры существовать не обязаны, например, если у группы лишь счетное число подгрупп.

Для заданной счетной группы $G$ один из первых вопросов состоит в том, существует ли непрерывная ИС-подгруппа. В конечном итоге хотелось бы описать структуру симплекса инвариантных вероятностных мер топологической динамической системы $(\operatorname{Inn}(G), S(G))$, где $\operatorname{Inn}(G)$ - группа внутренних автоморфизмов группы $G$, действующая на $S(G)$. Особый интерес представляют эргодические меры, т. е. крайние точки этого симплекса.

Более общая задача состоит в описании симплекса инвариантных вероятностных мер системы $(\Phi, S(G))$, где $\Phi$ - подгруппа группы $\operatorname{Aut}(G)$ автоморфизмов

*Первый и второй авторы поддержаны грантом NSF №DMS-1207699. Второй и третий авторы поддержаны Швейцарским национальным фондом научных исследований SNSF. 
группы $G$ (см. [1], [5], [27]). С этой задачей близко связано исследование инвариантных мер на пространстве корневых графов Шрейера группы $G$, на котором $G$ действует заменой корня. Эта точка зрения представлена в работах [14], [28].

Весьма плодотворная идея принадлежит здесь А. М. Вершику, который ввел понятие вполне несвободного действия локально компактной группы $G$ на пространстве $X$ с инвариантной мерой $\mu$, т. е. действия, обладающего тем свойством, что различные точки $x \in X \mu$-п.н. имеют различные стабилизаторы $\operatorname{St}_{G}(x)$. Тогда отображение St: $X \rightarrow S(G)$, задаваемое формулой $x \mapsto \operatorname{St}_{G}(x)$, $\mu$-п. н. инъективно. Образом меры $\mu$ при этом отображении является инвариантная относительно действия группы $G$ сопряжениями вероятностная мера на $S(G)$ (т. е. ИС-подгруппа), которая будет эргодична и непрерывна тогда же, когда и $\mu$. В работе [27] Вершик показал, что вполне несвободное действие группы $G$ определяет не только ИС-подгруппу, но и факторпредставление группы $G$. Он также воплотил описанный выше план в жизнь и описал все эргодические $\operatorname{Aut}(G)$-инвариантные меры на $S(G)$ для случая, когда $G$ - бесконечная симметрическая группа, см. [26], [27].

Боуэн [5] показал, что в свободной неабелевой группе $F_{r}, r \geqslant 2$, есть целый «зверинец» разных эргодических непрерывных ИС-подгрупп и что симплекс ИС-подгрупп п.н. бесконечного индекса в этом случае является симплексом Полсена. (Симплекс называется симплексом Полсена, если его крайние точки образуют плотное множество. Такой симплекс единствен с точностью до аффинного изоморфизма в силу результатов из [17].) Как показано в [4], уже у группы мигающих лампочек $\mathscr{L}=\mathbb{Z}_{2}$ l $\mathbb{Z}$ (простейшей конечно порожденной группы с $2^{\aleph_{0}}$ подгруппами) имеется симплекс Полсена ИС-подгрупп. Для заданной сюръекции $\phi: G \rightarrow H$ имеется естественный гомеоморфизм $\tilde{\phi}: S(H) \rightarrow S(G, \operatorname{Ker}(\phi))$ на подпространство $S(G, \operatorname{Ker}(\phi)) \subset S(G)$, состоящее из подгрупп группы $G$, содержащих ядро $\operatorname{Ker}(\phi)$. Это позволяет поднять любую ИС-подгруппу из $H$ в группу $G$, получая таким образом широкий спектр ИС-подгрупп группы $G$ из соответствующего множества ИС-подгрупп группы $H$. В частности, это соображение можно применить к накрывающей группу $\mathscr{L}$ свободной группе $F_{2}$.

$\mathrm{У}$ конечно порожденной почти нильпотентной группы лишь счетное число подгрупп и, следовательно, нет непрерывных ИС-подгрупп. По теореме Громова класс конечно порожденных почти нильпотентных групп совпадает с классом групп полиномиального роста. Напомним, что для заданной конечно порожденной группы $G$ с системой образующих $S$ можно рассмотреть ее функцию роста $\gamma(n)=\gamma_{(G, S)}(n)$, считающую число элементов длины не больше $n$. Тип роста этой функции при $n \rightarrow \infty$ не зависит от выбора множества образующих $S$ и может быть полиномиальным, экспоненциальным или промежуточным. Вопрос о существовании групп промежуточного роста был поставлен Милнором в [19] и решен вторым автором в [12]. Его основная конструкция сопоставляет каждой последовательности $\omega \in \Omega=\{0,1,2\}^{\mathbb{N}}$ группу $G_{\omega}$, порожденную четырьмя инволюциями $a_{\omega}, b_{\omega}, c_{\omega}, d_{\omega}$, и если последовательность $\omega$ не является финально постоянной, то $G_{\omega}$ - группа промежуточного роста. Более того, в работе [12] было замечено, что группы $G_{\omega}$ лежат в классе минимально бесконечных ветвящихся групп. Группа называется минимально бесконечной, если она бесконечна, но каждая ее собственная факторгруппа конечна. Группа называется ветвящейся, если она обладает точным транзитивным на каждом уровне действием на 
сферически однородном корневом дереве, причем жесткий стабилизатор каждого уровня дерева есть подгруппа конечного индекса (точные определения будут даны в разд. 4.2). Минимально бесконечные ветвящиеся группы составляют один из трех классов, на которые естественно разбивается класс минимально бесконечных групп [13].

Будучи минимально бесконечными, группы $G_{\omega}$ имеют лишь счетное число факторгрупп. Поэтому возник вопрос о существовании групп промежуточного роста с $2^{\aleph_{0}}$ факторгруппами, ответ на который был дан в [11]. Основная идея состояла в том, чтобы взять подходящее подмножество $\Lambda \subset \Omega$ мощности $2^{\aleph_{0}}$ и рассмотреть группу $U_{\Lambda}$, определяемую как факторгруппа свободной группы $F_{4}$ по нормальной подгруппе $N$, представляющей собой пересечение нормальных подгрупп $N_{\omega}, \omega \in \Lambda$, где $G_{\omega}=F_{4} / N_{\omega}$. В данной работе мы развиваем эту идею, используя ИС-подгруппы на $G_{\omega}$ и поднимая их на $U_{\Lambda}$, чтобы доказать наш основной результат.

Ветвящиеся группы являются источником естественно возникающих вполне несвободных действий и тем самым примеров нетривиальных (непрерывных) ИС-подгрупп. Действительно, в работе [2] показано, что продолжение по непрерывности ветвящегося действия, заданного на сферически однородном дереве $T$, на его границу $\partial T$ вполне несвободно относительно равномерной вероятностной меры на $\partial T$. Оно даже экстремально несвободно, т. е. стабилизаторы различных точек различны. Равномерная вероятностная мера на $\partial Т$ эргодична и инвариантна. Группы $G_{\omega}$ действуют на бинарном корневом дереве ветвящимся образом. Поднимая равномерную меру на $S\left(G_{\omega}\right)$ и затем на $S\left(U_{\Lambda}\right)$, получаем набор ИС-подгрупп на $U_{\Lambda}$. Затем мы переходим к доказательству того факта, что полученные таким образом ИС-подгруппы различны. Эти рассуждения позволяют доказать нашу основную теорему.

Основная теорема. Существует конечно порожденная группа промежуточного роста с $2^{\aleph_{0}}$ различными непрерывными эргодическими ИС-подгруппамu.

Мы также исследуем некоторые дополнительные свойства групп вида $U_{\Lambda}$, $\Lambda \subset \Omega$, такие, как конечная представимость, ветвимость и самоподобность.

\section{§2. Пространство меченых групп и универсальные группы}

Определение $1 . k$-меченой группой называется пара $(G, S)$, где $G$ - группа, а $S=\left(s_{1}, \ldots, s_{k}\right)$ - упорядоченное множество ее элементов (не обязательно различных), такое, что множество $\left\{s_{1}, \ldots, s_{k}\right\}$ порождает $G$. Отображение $k$-меченой группы $(G, S)$ в $k$-меченую группу $(H, T)$ называется каноническим, если образом элемента $s_{i}$ является $t_{i}$ для всех $i=1, \ldots, k$. Если это отображение задает эпиморфизм, то он называется меченым эпиморфизмом, а $(H, T)$ называется меченым образом меченой группы $(G, S)$. Две $k$-меченые группы $(G, S)$ и $(H, T)$ называются эквивалентными, если каноническое отображение определяет изоморфизм между $G$ и $H$.

Пространство (классов эквивалентности) $k$-меченых групп будет обозначаться через $\mathscr{M}_{k}$. На этом пространстве имеется естественная топология, которая, например, задается следующей метрикой: расстояние между двумя $k$-мечеными группами $(G, S)$ и $(H, K)$ равно $2^{-m}$, где $m$ - наибольшее натуральное число, 
такое, что шары радиуса $m$ в графах Кэли групп $(G, S)$ и $(H, K)$ изоморфны (как ориентированные помеченные графы). В работе [12] было замечено, что $\mathscr{M}_{k}$, снабженное этой топологией, является компактным вполне несвязным пространством.

Можно также определить это пространство следующим способом. Пусть $F_{k}$ свободная группа ранга $k$ с множеством свободных образующих $\left\{x_{1}, \ldots, x_{k}\right\}$. Пусть $\mathscr{N}_{k}$ - множество всех нормальных подгрупп в $F_{k}$ с топологией, индуцированной топологией Тихонова на множестве $\mathscr{P}\left(F_{k}\right) \cong\{0,1\}^{F_{k}}$ всех подмножеств группы $F_{k}$. У этой топологии есть базис, состоящий из множеств вида $\mathscr{O}_{A, B}=\left\{N \triangleleft F_{k} \mid A \subset N, B \cap N=\varnothing\right\}$, где $A$ и $B$ - конечные подмножества в $F_{k}$. Меченой группе $(G, S) \in \mathscr{M}_{k}$ сопоставим ядро $N_{(G, S)} \in \mathscr{N}_{k}$ естественного отображения $\pi_{(G, S)}: F_{k} \rightarrow G$, переводящего $x_{i}$ в $s_{i}$. Такое сопоставление определяет гомеоморфизм пространств $\mathscr{M}_{k}$ и $\mathscr{N}_{k}$, зависящий от выбора базиса в $F_{k}$ (см. [6]). Мы будем пользоваться взаимозаменяемостью этих двух пространств.

Определение 2. Пусть $\mathscr{C}=\left\{\left(G_{i}, S_{i}\right) \mid i \in I\right\}-$ подмножество в $\mathscr{M}_{k}$, и пусть $N_{\mathscr{C}}=\bigcap_{i \in I} N_{\left(G_{i}, S_{i}\right)}$. Универсальной группой семейства $\mathscr{C}$ называется $k$-меченая группа $\left(U_{\mathscr{C}}, S_{\mathscr{C}}\right)$, где $U_{\mathscr{C}}=F_{k} / N_{\mathscr{C}}$ и $S_{\mathscr{C}}$ - образ базиса $\left\{x_{1}, \ldots, x_{k}\right\}$.

Группа $U_{\mathscr{C}}$ обладает следующим свойством универсальности: если $(H, T)-$ меченая группа, такая, что для каждого $i \in I$ каноническое отображение из $(H, T)$ в $\left(G_{i}, S_{i}\right)$ определяет гомоморфизм групп, то каноническое отображение из $(H, T)$ в $\left(U_{\mathscr{C}}, S_{\mathscr{C}}\right)$ также определяет гомоморфизм групп.

Определить универсальную группу можно также следующим образом.

Определение 3. Для $\mathscr{C}=\left\{\left(G_{i}, S_{i}\right) \mid i \in I\right\} \subset \mathscr{M}_{k}$ положим $S_{i}=\left(s_{1}^{i}, \ldots, s_{k}^{i}\right)$. Пусть $U_{\mathscr{C}}^{\text {diag }}$ - подгруппа (полного) прямого произведения $\prod_{i \in I} G_{i}$, порожденная элементами $s_{j}=\left(s_{j}^{i}\right)_{i \in I}, j=1, \ldots, k$. Тогда $k$-меченая группа $\left(U_{\mathscr{C}}^{\operatorname{diag}}, S_{\mathscr{C}}^{\text {diag }}\right)$ называется диагональной группой семейства $\mathscr{C}$.

Непосредственная проверка показывает, что группа $\left(U_{\mathscr{C}}^{\text {diag }}, S_{\mathscr{C}}^{\text {diag }}\right)$ эквивалентна (как меченая группа) универсальной группе $\left(U_{\mathscr{C}}, S_{\mathscr{C}}\right)$ из определения 2.

Предложение 1. Пусть $\mathscr{C} \subset \mathscr{M}_{k}$. Тогда меченые группъ $\left(U_{\mathscr{C}}, S_{\mathscr{C}}\right)$ u $\left(U_{\overline{\mathscr{C}}}, S_{\overline{\mathscr{C}}}\right)$, где $\overline{\mathscr{C}}$ обозначает замыкание множества $\mathscr{C}$ в $\mathscr{M}_{k}$, эквивалентны.

Доказательство. Нам нужно показать, что

$$
\bigcap_{(G, S) \in \mathscr{C}} N_{(G, S)}=\bigcap_{(G, S) \in \overline{\mathscr{C}}} N_{(G, S)} .
$$

Ясно, что правая часть содержится в левой. Предположим. что некоторый элемент $g \in F_{k}$ содержится в левой части, но не содержится в правой. Тогда существует меченая группа $(G, S) \in \overline{\mathscr{C}}$, такая, что $g \notin N_{(G, S)}$. Пусть $\left\{\left(G_{n}, S_{n}\right)\right\}_{n \geqslant 0}-$ последовательность в $\mathscr{C}$, сходящаяся к $(G, S)$. Поскольку $g$ принадлежит левой части, он содержится в каждом $N_{\left(G_{n}, S_{n}\right)}$ и, согласно определению топологии на $\mathscr{N}_{k}$, в $N_{(G, S)}$, что приводит к противоречию.

Для элемента $w \in F_{k}$, такого, что $w \neq 1$, положим $\mathscr{O}_{w}=\left\{N \triangleleft F_{k} \mid w \in N\right\}$.

Лемма 1. Пусть $H \leqslant F_{k}-$ nодгрупnа, u nусть $w_{1}, \ldots, w_{m} \in H, w_{i} \neq 1$ для любого $i$. Тогда существует элемент $w \in H$, такой, что $w \neq 1$ u $\bigcup_{i=1}^{m} \mathscr{O}_{w_{i}} \subset \mathscr{O}_{w}$. 
Доказательство. Доказательство проводится индукцией по $m$. Случай $m=1$ очевиден, так как можно взять $w=w_{1}$. Поэтому предположим, что $m>1$.

Случай 1: $\left[w_{1}, w_{2}\right]=1$ в $F_{k}$. Тогда существуют элементы $w \in F_{k}$ и $s, t \in \mathbb{Z}$, такие, что $w_{1}^{s}=w_{2}^{t}=w$ (все нетривиальные абелевы подгруппы в свободной группе являются циклическими). Поэтому $\mathscr{O}_{w_{1}} \cup \mathscr{O}_{w_{2}} \subset \mathscr{O}_{w}$, и мы можем применить предположение индукции, заменяя $\mathscr{O}_{w_{1}}$ и $\mathscr{O}_{w_{2}}$ на $\mathscr{O}_{w}$.

Случай $2:\left[w_{1}, w_{2}\right] \neq 1$ в $F_{k}$. Тогда можно заменить $\mathscr{O}_{w_{1}}$ и $\mathscr{O}_{w_{2}}$ на $\mathscr{O}_{\left[w_{1}, w_{2}\right]}$ и применить предположение индукции.

Предложение 2. Пусть $\mathscr{C} \subset \mathscr{M}_{k}-$ замкнутое подмножество, такое, что ни одна группа из $\mathscr{C}$ не содержит неабелевой свободной подгруппь. Тогда у универсальной группы $U_{\mathscr{C}}$ также нет неабелевых свободных подгрупп.

Доказательство. Пусть $\mathscr{C}=\left\{\left(G_{i}, S_{i}\right) \mid i \in I\right\}$. Пусть $a, b \in U_{\mathscr{C}}-$ два различных элемента, задаваемых как слова над множеством образующих $S_{\mathscr{C}}$. Пусть элементы $w_{a}, w_{b} \in F_{k}$ таковы, что $\pi_{(G, S)}\left(w_{a}\right)=a$ и $\pi_{(G, S)}\left(w_{b}\right)=b$. Для каждого $i \in I$, поскольку у $G_{i}$ нет (неабелевых) свободных подгрупп, существует нетривиальный элемент $w_{i} \in\left\langle w_{a}, w_{b}\right\rangle \leqslant F_{k}$, такой, что $\pi_{\left(G_{i}, S_{i}\right)}\left(w_{i}\right)=1$, т. е. $w_{i} \in N_{\left(G_{i}, S_{i}\right)}$. Поэтому $\left\{\mathscr{O}_{w_{i}}\right\}_{i \in I}$ - открытое покрытие множества $\mathscr{C}$. Поскольку $\mathscr{C}$ компактно, у этого покрытия есть конечное подпокрытие $\mathscr{O}_{w_{1}}, \ldots, \mathscr{O}_{w_{n}}$. По лемме 1 существует нетривиальный элемент $w \in\left\langle w_{a}, w_{b}\right\rangle$, такой, что $\mathscr{C} \subset \mathscr{O}_{w}$. Отсюда следует, что $w=1$ в $U_{\mathscr{C}}$.

\section{§3. 2-группы Григорчука ${ }^{1)}$}

Мы напомним здесь конструкцию из работы [12]. Отметим, что в исходной конструкции из [12] группы определялись как действующие сохраняющими меру преобразованиями единичного интервала. Тут мы определим их как группы автоморфизмов бинарного корневого дерева.

Пусть $\Omega=\{0,1,2\}^{\mathbb{N}}$ - пространство бесконечных последовательностей $\omega=$ $\omega_{1} \omega_{2} \ldots \omega_{n} \ldots$, где $\omega_{i} \in\{0,1,2\}$, снабженное естественной топологией произведения. Пусть $\tau$ - преобразование сдвига, т.е. если $\omega=\omega_{1} \omega_{2} \ldots \in \Omega$, то $\tau \omega=\omega_{2} \omega_{3} \ldots$ Пусть $T$ - бинарное корневое дерево, множество вершин которого отождествляется с множеством $\{0,1\}^{*}$ всех конечных двоичных слов, а ребра определяются стандартным образом: $E=\left\{(w, w x) \mid w \in\{0,1\}^{*}, x \in\{0,1\}\right\}$. Для каждого $\omega \in \Omega$ рассмотрим автоморфизмы $\left\{a, b_{\omega}, c_{\omega}, d_{\omega}\right\}$ дерева $T$, рекурсивно определенные следующим образом.

Для $v \in\{0,1\}^{*}$ полагаем

$$
\begin{aligned}
& a(0 v)=1 v \quad \text { и } \quad a(1 v)=0 v, \\
& b_{\omega}(0 v)=0 \beta\left(\omega_{1}\right)(v), \quad c_{\omega}(0 v)=0 \zeta\left(\omega_{1}\right)(v), \quad d_{\omega}(0 v)=0 \delta\left(\omega_{1}\right)(v), \\
& b_{\omega}(1 v)=1 b_{\tau \omega}(v), \quad c_{\omega}(1 v)=1 c_{\tau \omega}(v), \quad d_{\omega}(1 v)=1 d_{\tau \omega}(v),
\end{aligned}
$$

где

$$
\begin{array}{lll}
\beta(0)=a, & \zeta(0)=a, & \delta(0)=e, \\
\beta(1)=a, & \zeta(1)=e, & \delta(1)=a, \\
\beta(2)=e, & \zeta(2)=a, & \delta(2)=a,
\end{array}
$$

a $e$ - тождественный автоморфизм дерева $T$.

1) Первый и третий авторы настаивают на использовании этой стандартной терминологии. 
Для каждого $\omega \in \Omega$ пусть $G_{\omega}-$ подгруппа в $\operatorname{Aut}(T)$, порожденная множеством $S_{\omega}=\left\{a, b_{\omega}, c_{\omega}, d_{\omega}\right\}$, так что $\mathscr{G}=\left\{\left(G_{\omega}, S_{\omega}\right) \mid \omega \in \Omega\right\}$ - подмножество в $\mathscr{M}_{4}$. В работе [12] было отмечено, что если две последовательности $\omega, \eta \in \Omega$, не являющиеся финально постоянными, имеют длинное общее начало, то 4-меченые группы $\left(G_{\omega}, S_{\omega}\right)$ и $\left(G_{\eta}, S_{\eta}\right)$ близки друг к другу в $\mathscr{M}_{4}$. Было замечено также, что для финально постоянных последовательностей $\omega$ группы $\left(G_{\omega}, S_{\omega}\right)$ изолированы в $\left\{\left(G_{\omega}, S_{\omega}\right) \mid \omega \in \Omega\right\}$. Поэтому, удаляя из этого множества эти изолированные точки и переходя к его замыканию в $\mathscr{M}_{4}$, получаем компактное подмножество $\widetilde{\mathscr{G}}=\left\{\left(\widetilde{G}_{\omega}, \widetilde{S}_{\omega}\right) \mid \omega \in \Omega\right\} \subset \mathscr{M}_{4}$, гомеоморфное множеству $\Omega$ (и, следовательно, канторову множеству) при отображении $\omega \mapsto\left(\widetilde{G}_{\omega}, \widetilde{S}_{\omega}\right)$. Заметим, что $\left(\widetilde{G}_{\omega}, \widetilde{S}_{\omega}\right)=\left(G_{\omega}, S_{\omega}\right)$ тогда и только тогда, когда последовательность $\omega$ не финально постоянна, и что $\left(\widetilde{G}_{\omega}, \widetilde{S}_{\omega}\right)=\lim _{n \rightarrow \infty}\left(G_{\omega(n)}, S_{\omega(n)}\right)$, если $\omega$ финально постоянна, где $\left\{\omega^{(n)}\right\}_{n \geqslant 0}$ - последовательность не финально постоянных элементов из $\Omega$, сходящаяся к $\omega$ (предел не зависит от выбора последовательности $\left.\left\{\omega^{(n)}\right\}_{n \geqslant 0}\right)$. Другими словами, семейства $\mathscr{G}$ и $\widetilde{\mathscr{G}}$ отличаются только счетным числом точек.

Заметим, что справедливо следующее утверждение: $N_{\left(\widetilde{G}_{\omega}, \widetilde{S}_{\omega}\right)}=N_{\left(G_{\omega}, S_{\omega}\right)}$, если $\omega$ не финально постоянна, и $N_{\left(\widetilde{G}_{\omega}, \widetilde{S}_{\omega}\right)} \subset N_{\left(G_{\omega}, S_{\omega}\right)}$ для финально постоянного элемента $\omega \in \Omega$.

Пусть $\Omega_{\infty}$ - множество последовательностей из $\Omega$, в которых все три символа $\{0,1,2\}$ встречаются бесконечно много раз, и пусть $\Omega_{0}-$ множество финально постоянных последовательностей. О группах из $\mathscr{G}$ и $\widetilde{\mathscr{G}}$ известно следующее:

Теорема 1 [12]. 1. Все группь $G_{\omega}, \omega \in \Omega,-$ бесконечные финитно аппроксимируемые группы.

2. Группа $G_{\omega}$ содержит подгруппу конечного индекса, изоморфную $\mathbb{Z}^{2^{n}}$, если $\omega$ становится постоянной, начиная с $n$-й координаты.

3. Если $\omega \notin \Omega_{0}$, то рост группы $G_{\omega}$ является промежуточным между полиномиальным и экспоненииальным.

4. Если $\omega \in \Omega_{0}$, то группа $\widetilde{G}_{\omega}$ почти метабелева, бесконечно представима и имеет экспоненииальный рост.

5. Если $\omega \in \Omega_{\infty}$, mо $G_{\omega}-$ периодическая 2-группа.

6. Если $\omega \in \Omega_{\infty}$, то группа $G_{\omega}$ минимально бесконечна, т.е. все ее собственные факторгруппы конечны.

7. Пусть $\omega_{1}, \omega_{2} \in \Omega_{\infty}$. Изоморфизм $G_{\omega_{1}} \cong G_{\omega_{2}}$ имеет место тогда и только тогда, когда слово $\omega_{1}$ можно получить из $\omega_{2}$, посимвольно применяя некоторую перестановку из $\operatorname{Sym}(\{0,1,2\})$.

Доказательство. Доказательство пп. 1, 2, 3 и 5 содержится в теореме 2.1 из [12]. Пункт 4 доказан в [12, теоремы $6.1,6.2]$, а пункт 6 -в [12, теорема 8.1]. Пункт 7 доказан в [21, теорема 2.10.13].

\section{§4. Некоторые свойства полной универсальной группы $U$}

Про универсальные группы, отвечающие семействам $\mathscr{G}$ и $\widetilde{\mathscr{G}}$, известно следующее:

Предложение 3. $U_{\mathscr{G}}=U_{\widetilde{\mathscr{G}}}$. 
Доказательство. В обозначениях определения 2 нам нужно доказать следующее равенство:

$$
N_{\mathscr{G}}:=\bigcap_{\omega \in \Omega} N_{\left(G_{\omega}, S_{\omega}\right)}=N_{\tilde{\mathscr{G}}}=: \bigcap_{\omega \in \Omega} N_{\left(\tilde{G}_{\omega}, \tilde{S}_{\omega}\right)} .
$$

Так как $N_{\left(\widetilde{G}_{\omega}, \widetilde{S}_{\omega}\right)} \subset N_{\left(G_{\omega}, S_{\omega}\right)}$ при каждом $\omega \in \Omega$, то правая часть этого равенства содержится в левой. Поскольку множество $\left\{\left(\widetilde{G}_{\omega}, \widetilde{S}_{\omega}\right) \mid \omega \in \Omega \backslash \Omega_{0}\right\}$ плотно в $\widetilde{\mathscr{G}}$, из предложения 1 вытекает, что

$$
N_{\tilde{\mathscr{G}}}=\bigcap_{\omega \in \Omega \backslash \Omega_{0}} N_{\left(\tilde{G}_{\omega}, \tilde{S}_{\omega}\right)}
$$

Таким образом,

$$
N_{\mathscr{G}} \subset \bigcap_{\omega \in \Omega \backslash \Omega_{0}} N_{\left(G_{\omega}, S_{\omega}\right)}=\bigcap_{\omega \in \Omega \backslash \Omega_{0}} N_{\left(\tilde{G}_{\omega}, \tilde{S}_{\omega}\right)}=N_{\widetilde{\mathscr{G}}}
$$

Мы будем использовать обозначение $U=U_{\mathscr{G}}$ для полной универсальной группы и обозначим через $S=\{a, b, c, d\}$ ее канонические образующие. Отметим, что в $U$ выполняются основные соотношения $a^{2}=b^{2}=c^{2}=d^{2}=b c d=1$.

Теорема 2. Группа $U$ не содержит неабелевых свободных подгрупп, имеет равномерно экспоненциальный рост и не конечно представима.

Доказательство. Поскольку все группы в $\widetilde{\mathscr{G}}$ аменабельны (и потому не могут иметь неабелевых свободных подгрупп), первое утверждение следует из предложения 2. Согласно п. 4 теоремы 1 , группа $\tilde{G}_{\eta}$, где $\eta=000 \ldots$, является элементарной аменабельной группой экспоненциального роста и, следовательно, равномерно экспоненциального роста в силу результатов из [23]. Таким образом, $U$ - группа равномерно экспоненциального роста. По теореме 1.10 из [3] каждая конечно представимая группа, отображающаяся на группы $G_{\omega}, \omega \in \Omega$, должна быть большой, т. е. иметь подгруппу конечного индекса, отображающуюся на неабелеву свободную группу. В частности, такая группа содержит неабелеву свободную подгруппу. Поэтому $U$ не может быть конечно представимой.

4.1. $U$ как автоматная группа. В этом разделе мы реализуем $U$ как автоматную группу и исследуем ее дальнейшие свойства. Прежде всего напомним некоторые базовые факты о группах автоморфизмов корневых деревьев.

Пусть $T_{d}$ - регулярное корневое дерево степени $d$ с множеством вершин $\{0,1,2, \ldots, d-1\}^{*}$. Сечением автоморфизма $g \in \operatorname{Aut}\left(T_{d}\right)$ в $x \in\{0,1, \ldots, d-1\}$ называется автоморфизм $g_{x}$ дерева $T_{d}$, однозначно определенный соотношениeM

$$
g(x v)=g(x) g_{x}(v) \quad \text { для всех } v \in\{0,1, \ldots, d-1\} .
$$

Отсюда получается изоморфизм

$$
\begin{aligned}
\operatorname{Aut}\left(T_{d}\right) & \rightarrow S_{d} \ltimes\left(\operatorname{Aut}\left(T_{d}\right) \times \cdots \times \operatorname{Aut}\left(T_{d}\right)\right), \\
g & \mapsto \sigma_{g}\left(g_{0}, \ldots, g_{d-1}\right),
\end{aligned}
$$

где $\sigma_{g}$ описывает, каким образом $g$ переставляет поддеревья первого уровня, а автоморфизмы $g_{i}$ описывают действие автоморфизма $g$ внутри каждого из этих поддеревьев. (Здесь $S_{d}$ - симметрическая группа на $d$ символах.) 
Определение 4. Подгруппа $G \leqslant \operatorname{Aut}\left(T_{d}\right)$ называется самоподобной, если $g_{x} \in G$ для любых $g \in G$ и $x \in\{0,1, \ldots, d-1\}$.

Обзор теории самоподобных групп и смежных с ней тем можно найти в [15], [21].

Стандартный способ построения самоподобных групп состоит в том, чтобы задать списки символов $S=\left\{s^{1}, \ldots, s^{m}\right\}$ и перестановок $\sigma_{1}, \ldots, \sigma_{m} \in S_{d}$ и рассмотреть систему

$$
\begin{gathered}
s^{1}=\sigma_{1}\left(s_{0}^{1}, \ldots, s_{d-1}^{1}\right), \\
\ldots \ldots \ldots \ldots \ldots \ldots \\
s^{m}=\sigma_{m}\left(s_{0}^{m}, \ldots, s_{d-1}^{m}\right),
\end{gathered}
$$

где $s_{j}^{i} \in S$. Если $\sigma_{i}=\mathrm{id}$, то этот символ будет опускаться в записи $s^{i}$. Такая система определяет единственное множество из $m$ автоморфизмов дерева $T_{d}$. Очевидно, что группа $G=\langle S\rangle$ будет самоподобной. Поскольку множество образующих $S$ в этом случае замкнуто относительно взятия сечений, действие этой группы можно моделировать автоматом типа Мили, в котором каждому образующему будет соответствовать состояние автомата (см., например, рисунок на следующей странице). Такие группы, т. е. группы, порожденные состояниями автомата типа Мили, называются автоматными группами. Детальное обсуждение автоматных групп имеется в работе [9].

Рассмотрим дерево $T_{6}$, определенное алфавитом

$$
\mathscr{A}=\{0,1\} \times\{0,1,2\}=\{(0,0),(0,1),(0,2),(1,0),(1,1),(1,2)\},
$$

символы которого занумерованы числами $0,1, \ldots, 5$. Пусть $V \leqslant \operatorname{Aut}\left(T_{6}\right)$ порождена следующими элементами $A, B, C, D$ :

$$
\begin{array}{ll}
A=(14)(25)(36) & (E, E, E, E, E, E), \\
B= & (A, A, E, B, B, B), \\
C= & (A, E, A, C, C, C), \\
D= & (E, A, A, D, D, D) .
\end{array}
$$

Здесь $(14)(25)(36)$ - элемент симметрической группы $S_{6}$, а $E$ соответствует тождественному автоморфизму. Заметим, что $A^{2}=B^{2}=C^{2}=D^{2}=B C D=1$. Соответствующий автомат изображен на следующей странице.

Покажем, что группа $V$ изоморфна $U$ (как меченая группа).

Для заданных $\omega \in \Omega$ и $u \in\{0,1\}^{*}$ пусть $\omega^{u} \in\{0,1,2\}^{*}$ - начальная часть длины $|u|$ слова $\omega$. Заметим, что $\omega^{u v}=\omega^{u}\left(\tau^{|u|}(\omega)\right)^{v}$ для всех $u, v \in\{0,1\}^{*}$.

Для любого $\omega \in \Omega$ пусть $T_{\omega}=\left\{(u, v) \in T_{6} \mid u \in\{0,1\}^{*}, v=\omega^{u}\right\}$. Очевидно, что $T_{\omega}$ - бинарное поддерево дерева $T_{6}$. Обозначим $\{0,1\}^{*}$ через $T_{2}$ и определим отображение $\phi_{\omega}: T_{\omega} \rightarrow T_{2}$ формулой $\phi_{\omega}(u, v)=u$, которая, очевидно, задает биекцию. При $(u, v) \in T_{\omega}$ и $\left(u^{\prime}, v^{\prime}\right) \in T_{\tau^{|u|} \mid \omega}$ имеем

$$
\phi_{\omega}\left(u v, u^{\prime} v^{\prime}\right)=\phi_{\omega}(u, v) \phi_{\tau|u| \omega}\left(u^{\prime}, v^{\prime}\right) .
$$

Для заданных $g \in V$ и $\omega \in \Omega$ определим гомоморфизм групп $\psi_{\omega}: V \rightarrow$ $\operatorname{Aut}\left(T_{2}\right)$ формулой $\psi_{\omega}(g)(u)=\phi_{\omega}\left(g\left(u, \omega^{u}\right)\right)$ для всех $u \in T_{2}$. Непосредственная проверка показывает, что $\psi_{\omega}(g) \in \operatorname{Aut}\left(T_{2}\right)$ и что $\psi_{\omega}$ - гомоморфизм групп. 


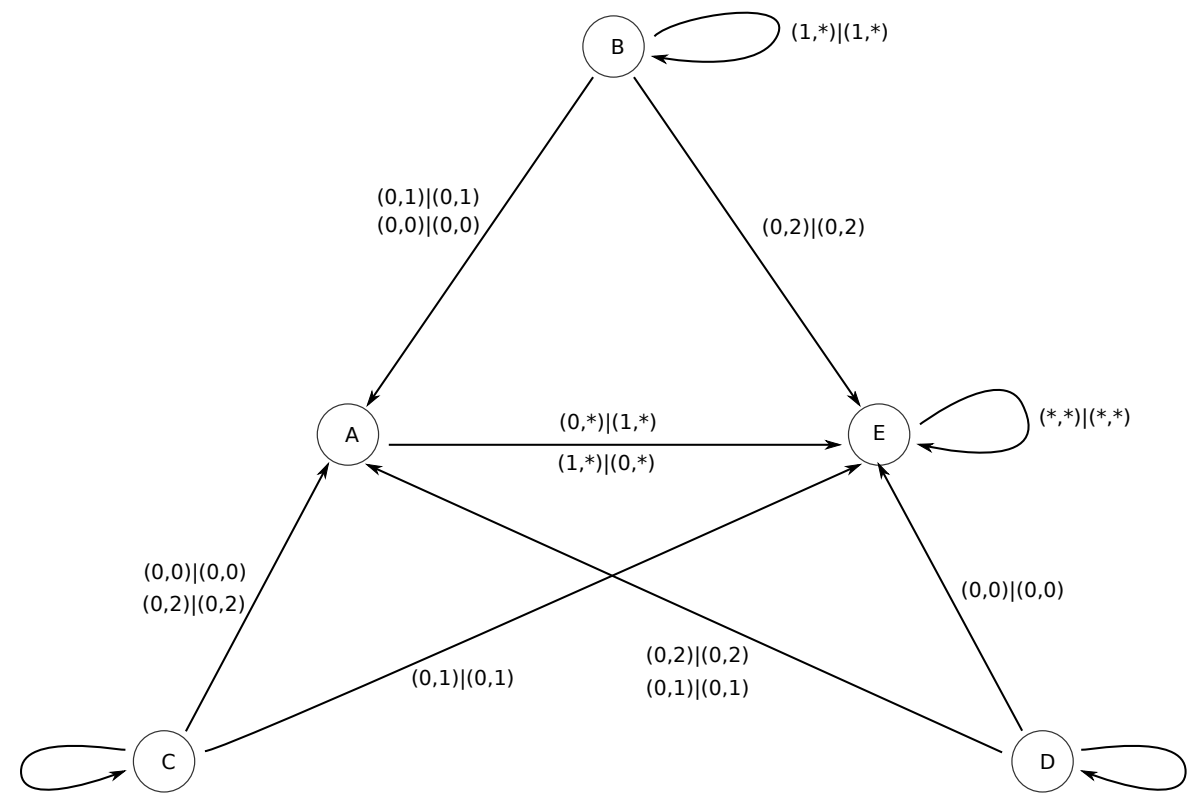

$(1, *) \mid(1, *)$

$(1, *) \mid(1, *)$

Лемма 2. Для всех $u \in T_{2}$, таких, что $|u|=n$, выполнено равенство

$$
\psi_{\omega}(g)_{u}=\psi_{\tau^{n} \omega}\left(g_{\left(u, \omega^{u}\right)}\right) .
$$

Доказательство. Пусть $u, z \in\{0,1\}^{*}$ и $|u|=n$. Положим $\omega^{u}=v$ и $\left(\tau^{n} \omega\right)^{z}$ $=v^{\prime}$; тогда $g\left(u z, \omega^{u z}\right)=g\left(u z, v v^{\prime}\right)=g\left((u, v)\left(z, v^{\prime}\right)\right)=g(u, v) g_{(u, v)}\left(z, v^{\prime}\right)$. Поэтому, согласно (2),

$$
\begin{aligned}
\psi_{\omega}(g)(u z) & =\phi_{\omega}\left(g\left(u z, \omega^{u z}\right)\right)=\phi_{\omega}\left(g(u, v) g_{(u, v)}\left(z, v^{\prime}\right)\right) \\
& =\phi_{\omega}(g(u, v)) \phi_{\tau^{n} \omega}\left(g_{(u, v)}\left(z, v^{\prime}\right)\right)=\psi_{\omega}(g)(u) \psi_{\tau^{n} \omega}\left(g_{(u, v)}\right)(z) .
\end{aligned}
$$

Отсюда вытекает нужный результат.

Лемма 3. Для любого $\omega \in \Omega$ гомоморфизм $\psi_{\omega}$ определяет меченый сюорективный гомоморфизм $\psi_{\omega}: V \rightarrow G_{\omega}$.

Доказательство. Достаточно показать, что $\psi_{\omega}$ переводит образующие группы $V$ в образующие группы $G_{\omega}$. Прежде всего, по определению элемента $A$ имеем

$$
\psi_{\omega}(A)(u)=\phi_{\omega}\left(A\left(u, \omega^{u}\right)\right)=\phi_{\omega}\left(\left(a(u), \omega^{u}\right)\right)=a(u) \quad \text { для всех } u .
$$

Покажем индукцией по $|u|$, что $B, C, D$ отображаются соответственно в $b_{\omega}$, $c_{\omega}, d_{\omega}$. Для $|u|=1$ это показывается непосредственно. Используя лемму 2 и предположение индукции, для $u \in\{0,1\}^{*}$ имеем

$\psi_{\omega}(B)(0 u)=0 \psi_{\omega}(B)_{0}(u)=0 \psi_{\omega}\left(B_{\left(0, \omega^{0}\right)}\right)(u)=\left\{\begin{array}{ll}0 a(u), & \text { если } \omega^{0}=0,1, \\ 0 u, & \text { если } \omega^{0}=2\end{array}=b_{\omega}(0 u)\right.$.

Аналогичным образом можно показать, что $\psi_{\omega}(B)(1 u)=b_{\omega}(1 u)$ для всех $u \in$ $\{0,1\}^{*}$, так что $\psi_{\omega}(B)=b_{\omega}$. Повторяя рассуждение, показываем, что $\psi_{\omega}(C)=$ $c_{\omega}, \psi_{\omega}(D)=d_{\omega}$. 
Теорема 3. Группа $V$ изоморфна универсальной группе $U$ (как меченая групnа).

Доказательство. По лемме 3 для каждого $\omega \in \Omega$ существует меченая сюръекция $\psi_{\omega}: V \rightarrow G_{\omega}$, так что существует и меченая сюръекция $\psi: V \rightarrow U$. Если элемент $g \in V$ нетривиален, возьмем вершину $v \in T_{6}$, такую, что $g v \neq v$. Пусть $\omega \in \Omega$ таков, что $v \in T_{\omega}$. Из этого вытекает, что $\psi_{\omega}(g) \neq 1$ и, следовательно, $\psi(g) \neq 1$. Таким образом, $\psi$ - меченый изоморфизм.

Всюду далее мы отождествляем $U$ и $V$.

В работе [25] Сидки привел классификацию автоматных групп в соответствии с их «ростом активности» и предположил, что автоматные группы полиномиального роста аменабельны. Отметим, что автомат, определяющий группу $U$, имеет экспоненциальный рост активности в классификации Сидки. Вопрос об аменабельности группы $U$ остается открытым. Заметка [20], в которой утверждается, что она аменабельна, к сожалению, содержит ошибку.

4.2. Структура ветвления группы $\boldsymbol{U}$. Пусть $G$-группа, действующая на $d$-регулярном корневом дереве $T_{d}$. Для вершины $v$ дерева $T_{d}$ обозначим через $T_{v}$ поддерево с корнем в $v$, а носитель элемента $g \in G$, т. е. множество вершин, не являющихся неподвижными для $g$, обозначим через $\operatorname{supp}(g)$. Стабилизатором вершины $v$ называется подгруппа $\operatorname{St}_{G}(v)=\{g \in G \mid g(v)=v\}$. Жестким стабилизатором вершины $v$ называется подгруппа $\operatorname{Rist}_{G}(v)=\{g \in$ $\left.G \mid \operatorname{supp}(g) \subset T_{v}\right\}$. Жестким стабилизатором уровня $n$ называется подгруппа $\operatorname{Rist}_{G}(n)=\left\langle\operatorname{Rist}_{G}(v)|| v \mid=n\right\rangle$. Поскольку жесткие стабилизаторы различных вершин одного и того же уровня коммутируют, справедливо равенство $\operatorname{Rist}_{G}(n)=\prod_{|v|=n} \operatorname{Rist}_{G}(v)$.

Определение 5. Пусть $G$ - группа автоморфизмов корневого дерева $T$. Говорят, что $G$ - почти ветвящаяся группа (слабо почти ветвящаяся группа), если подгруппа $\operatorname{Rist}_{G}(n)$ имеет в $G$ конечный индекс (нетривиальна) для всех $n \geqslant 1$. Если к тому же $G$ действует транзитивно на каждом уровне дерева, то она называется ветвящейся группой (слабо ветвящейся группой).

Класс (слабо) ветвящихся групп интересен с разных точек зрения и играет важную роль в классификации минимально бесконечных групп, т. е. групп, все собственные гомоморфные образы которых конечны (см. [13] по поводу детального описания ветвящихся и минимально бесконечных групп).

Упомянем следующий факт, который будет использован в дальнейшем (где также будет приведено и его альтернативное доказательство).

Теорема 4 [12]. Для $\omega \in \Omega_{\infty}$ группа $G_{\omega}$ ветвящаяся.

Отметим, что термин «ветвящаяся группа» в статье [12] не использовался.

Если $G$ - самоподобная группа, то стандартный метод доказательства того, что она почти ветвящаяся (слабо почти ветвящаяся) состоит в том, чтобы найти подгруппу $K$ конечного индекса (нетривиальную подгруппу) в $G$, такую, что ее образ $\phi(K)$ содержит подгруппу $K \times \cdots \times K$, где $\phi: \operatorname{Aut}\left(T_{d}\right) \rightarrow S_{d} \ltimes\left(\operatorname{Aut}\left(T_{d}\right) \times\right.$ $\left.\cdots \times \operatorname{Aut}\left(T_{d}\right)\right)$ определяется так же, как в предыдущем разделе. Это включение обозначается через $K \succcurlyeq K \times \cdots \times K$, и в таком случае говорят, что группа $G$ является регулярно (слабо) почти ветвящейся группой над подгруппой $K$.

Определение 6. Пусть $G$-самоподобная группа автоморфизмов $d$-регулярного корневого дерева $T_{d}$. Говорят, что $G$ самовоспроизводящаяся, если для 
любых $g \in G$ и $x \in\{0,1, \ldots, d-1\}$ существует элемент $h \in \operatorname{St}_{G}(1)$, такой, что $h_{x}=g$.

О действии $U$ на $T_{6}$ известно следующее:

Теорема 5. Группа U является самовоспроизводящейся регулярно слабо почти ветвящейся над своим третъим коммутантом группой.

Доказательство. Заметим, что стабилизатор $\operatorname{St}_{U}(1)$ порожден элементами $\{b, c, d, a b a, a c a, a d a\}$. Поскольку

$$
\begin{aligned}
b & =(a, a, 1, b, b, b), \quad c & =(a, 1, a, c, c, c), \quad d & =(1, a, a, d, d, d), \\
a b a & =(b, b, b, a, a, 1), \quad a c a & =(c, c, c, a, 1, a), \quad a d a & =(d, d, d, 1, a, a),
\end{aligned}
$$

группа $U$ самовоспроизводящаяся.

Мы утверждаем, что коммутант $U^{\prime}$ порождается элементами $(a b)^{2},(a c)^{2}$, $(a d)^{2}$. Из основных соотношений следует, что $a, b, c, d$ - элементы порядка 2 и что $b, c$ и $d$ коммутируют друг с другом. Поэтому $U^{\prime}$ порождается $\kappa a \kappa$ нормальная подгруппа элементами

$$
[a, b]=(a b)^{2}, \quad[a, c]=(a c)^{2}, \quad[a, d]=(a d)^{2} .
$$

Таким образом, достаточно показать, что подгруппа, порожденная элементами $(a b)^{2},(a c)^{2},(a d)^{2}$, нормальна в $U$. Очевидно, сопряжение элементом а обращает элементы $(a b)^{2},(a c)^{2},(a d)^{2}$. Для других сопряжений получаем, используя соотношение $b c d=1$, что

$$
x(a x)^{2} x=(x a)^{2}=\left((a x)^{2}\right)^{-1} \quad \text { и } \quad y(a x)^{2} y=(y a)^{2}(a z)^{2}=\left((a y)^{2}\right)^{-1}(a z)^{2},
$$

где элементы $x, y, z \in\{b, c, d\}$ попарно различны. Таким образом, $U^{\prime}$ порождается элементами $(a b)^{2},(a c)^{2},(a d)^{2}$.

Далее, мы утверждаем, что $U$ является почти слабо ветвящейся над третьим коммутантом $U^{\prime \prime \prime}$, т. е. $U^{\prime \prime \prime} \succcurlyeq U^{\prime \prime \prime} \times U^{\prime \prime \prime} \times U^{\prime \prime \prime} \times U^{\prime \prime \prime} \times U^{\prime \prime \prime} \times U^{\prime \prime \prime}$. Пусть

$$
t=\left[(a b)^{2},(a c)^{2}\right], \quad v=\left[(a b)^{2},(a d)^{2}\right], \quad w=\left[(a c)^{2},(a d)^{2}\right] .
$$

Второй коммутант $U^{\prime \prime}$ порождается как нормальная подгруппа элементами $t$, $v$ и $w$. Поэтому $U^{\prime \prime}$ порождается множеством $\left\{t^{g_{1}}, v^{g_{2}}, w^{g_{3}} \mid g_{i} \in U\right\}$. Отсюда следует, что $U^{\prime \prime \prime}$ порождается как нормальная подгруппа множеством

$$
S=\left\{\left[t^{g_{1}}, v^{g_{2}}\right],\left[t^{g_{3}}, w^{g_{4}}\right],\left[v^{g_{5}}, w^{g_{6}}\right] \mid g_{i} \in U\right\} .
$$

Справедливы равенства

$$
\begin{aligned}
& h_{1}=\left[\left[(a b)^{2}, b\right],\left[b,(c a)^{2}\right]\right]=(t, *, 1,1,1,1), \\
& h_{2}=\left[\left[(a b)^{2}, b\right],\left[c,(d a)^{2}\right]\right]=(v, 1,1,1, *, 1), \\
& h_{3}=\left[\left[c,(c a)^{2}\right],\left[b,(d a)^{2}\right]\right]=(w, 1,1,1,1, *), \\
& h_{4}=\left[\left[b,(b a)^{2}\right],\left[d,(c a)^{2}\right]\right]=(1, t, 1, *, 1,1), \\
& h_{5}=\left[\left[d,(a d)^{2}\right],\left[b,(b a)^{2}\right]\right]=(1, v, 1,1, *, 1), \\
& h_{6}=\left[\left[d,(c a)^{2}\right],\left[b,(d a)^{2}\right]\right]=(1, w, 1,1,1, *), \\
& h_{7}=\left[\left[c,(b a)^{2}\right],\left[d,(c a)^{2}\right]\right]=(1,1, t, *, 1,1), \\
& h_{8}=\left[\left[d,(b a)^{2}\right],\left[c,(d a)^{2}\right]\right]=(1,1, v, 1, *, 1), \\
& h_{9}=\left[\left[c,(c a)^{2}\right],\left[d,(d a)^{2}\right]\right]=(1,1, w, 1,1, *),
\end{aligned}
$$


где звездочкой * обозначены не играющие роли элементы группы $U$. (Их удобнее всего проверить с помощью пакета GAP, http://www.gap-system.org/ Packages/automgrp.html.) Очевидно, что $h_{i} \in U^{\prime \prime}$ при всех $i=1,2,3,4,5,6$. Так как $U$ - самовоспроизводящаяся группа, то для заданных $g_{1}, g_{2}, g_{3}, g_{4}, g_{5}, g_{6} \in U$ существуют элементы $\gamma_{1}, \gamma_{2}, \gamma_{3}, \gamma_{4}, \gamma_{5}, \gamma_{6} \in U$, такие, что

$$
\begin{aligned}
& \gamma_{1}=\left(g_{1}, *, *, *, *, *\right), \quad \gamma_{2}=\left(g_{2}, *, *, *, *, *\right), \quad \gamma_{3}=\left(g_{3}, *, *, *, *, *\right), \\
& \gamma_{4}=\left(g_{4}, *, *, *, *, *\right), \quad \gamma_{5}=\left(g_{5}, *, *, *, *, *\right), \quad \gamma_{6}=\left(g_{6}, *, *, *, *, *\right) .
\end{aligned}
$$

Таким образом,

$$
\begin{aligned}
& {\left[h_{1}^{\gamma_{1}}, h_{2}^{\gamma_{2}}\right]=\left(\left[t^{g_{1}}, v^{g_{2}}\right], 1,1,1,1,1\right),} \\
& {\left[h_{1}^{\gamma_{3}}, h_{3}^{\gamma_{4}}\right]=\left(\left[t^{g_{3}}, w^{g_{4}}\right], 1,1,1,1,1\right),} \\
& {\left[h_{2}^{\gamma_{5}}, h_{3}^{\gamma_{6}}\right]=\left(\left[v^{g_{5}}, w^{g_{6}}\right], 1,1,1,1,1\right),}
\end{aligned}
$$

и левые части этих равенств, очевидно, принадлежат $U^{\prime \prime \prime}$. Так как $U$ самовоспроизводящаяся, то

$$
U^{\prime \prime \prime} \succcurlyeq U^{\prime \prime \prime} \times 1 \times 1 \times 1 \times 1 \times 1 .
$$

Проделывая то же самое со второй и третьей координатами и используя другие $h_{i}$, видим, что

$$
\begin{aligned}
& U^{\prime \prime \prime} \succcurlyeq 1 \times U^{\prime \prime \prime} \times 1 \times 1 \times 1 \times 1, \\
& U^{\prime \prime \prime} \succcurlyeq 1 \times 1 \times U^{\prime \prime \prime} \times 1 \times 1 \times 1 ;
\end{aligned}
$$

сопрягая, наконец, элементом $a$, получаем также, что

$$
\begin{aligned}
& U^{\prime \prime \prime} \succcurlyeq 1 \times 1 \times 1 \times U^{\prime \prime \prime} \times 1 \times 1, \\
& U^{\prime \prime \prime} \succcurlyeq 1 \times 1 \times 1 \times 1 \times U^{\prime \prime \prime} \times 1, \\
& U^{\prime \prime \prime} \succcurlyeq 1 \times 1 \times 1 \times 1 \times 1 \times U^{\prime \prime \prime},
\end{aligned}
$$

откуда видно, что

$$
U^{\prime \prime \prime} \succcurlyeq U^{\prime \prime \prime} \times U^{\prime \prime \prime} \times U^{\prime \prime \prime} \times U^{\prime \prime \prime} \times U^{\prime \prime \prime} \times U^{\prime \prime \prime} .
$$

Очевидно, что $U^{\prime \prime \prime}$ нетривиальна, так как у группы $U$ есть неразрешимые факторгруппы.

Заметим, что группа $U / U^{\prime \prime \prime}$ отображается на группу $\widetilde{G}_{000 . .}$ и потому бесконечна. Далее, $U$ не обладает ветвящимися действиями (ни на каком корневом дереве), поскольку, как доказано в [13], все нетривиальные факторгруппы ветвящихся групп почти абелевы.

4.3. Структура ветвления общих универсальных групп. В этом разделе исследуется структура ветвления произвольных универсальных групп.

Для $\omega \in \Omega$ определена инъекция

$$
\begin{aligned}
\phi_{\omega}: G_{\omega} & \rightarrow \quad S_{2} \ltimes\left(G_{\tau \omega} \times G_{\tau \omega}\right), \\
a & \mapsto((01) ;(1,1)), \\
b_{\omega} & \mapsto \quad\left(1 ;\left(\beta\left(\omega_{0}\right), b_{\tau \omega}\right)\right), \\
c_{\omega} & \mapsto \quad\left(1 ;\left(\zeta\left(\omega_{0}\right), c_{\tau \omega}\right)\right), \\
d_{\omega} & \mapsto \quad\left(1 ;\left(\delta\left(\omega_{0}\right), d_{\tau \omega}\right)\right) .
\end{aligned}
$$


Для подгрупп $H \leqslant G_{\omega}$ и $H \leqslant G_{\tau \omega}$ будем писать $K \times K \preceq H$, если $K \times$ $K \leqslant \phi_{\omega}(H)$. Заметим, что в таком случае $H$ содержит подгруппу, изоморфную $K \times K$.

Предложение 4. Для $\omega \in \Omega$ выполнено соотношение $G_{\tau \omega}^{\prime \prime \prime} \times G_{\tau \omega}^{\prime \prime \prime} \preceq G_{\omega}^{\prime \prime \prime}$.

Доказательство. Предположим, что $\omega_{0}=0$. Определим отображение

$$
\pi: U \times U \times U \times U \times U \times U \rightarrow G_{\omega} \times G_{\omega},
$$

полагая $\pi\left(u_{1}, u_{2}, u_{3}, u_{4}, u_{5}, u_{6}\right)=\left(\psi_{\omega}\left(u_{1}\right), \psi_{\omega}\left(u_{4}\right)\right)$, где $\psi_{\omega}$ определено в разд. 4.1. Пусть $\phi: U \rightarrow S_{6} \ltimes U \times U \times U \times U \times U \times U$ - каноническое отображение.

Тогда имеется коммутативная диаграмма

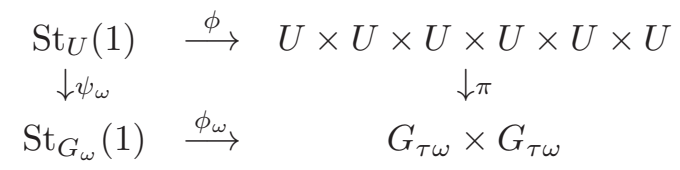

По теореме 5 имеем $U^{\prime \prime \prime} \times U^{\prime \prime \prime} \times U^{\prime \prime \prime} \times U^{\prime \prime \prime} \times U^{\prime \prime \prime} \times U^{\prime \prime \prime} \preceq U^{\prime \prime \prime}$. Так как $\psi_{\omega}\left(U^{\prime \prime \prime}\right)=$ $G_{\omega}^{\prime \prime \prime}$, то $G_{\tau \omega}^{\prime \prime \prime} \times G_{\tau \omega}^{\prime \prime \prime} \preceq G_{\omega}^{\prime \prime \prime}$.

Случаи, когда $\omega_{0}=1$ или $\omega_{0}=2$, можно рассмотреть аналогичным образом, изменяя $\pi$.

Следствие 1. При $\omega \in \Omega_{\infty}$ группа $G_{\omega}$ является ветвящейся группой.

Доказательство. Из предложения 4 по индукции вытекает, что

$$
\prod_{1}^{2^{n}} G_{\tau^{n} \omega}^{\prime \prime \prime} \preceq G_{\omega}^{\prime \prime \prime}
$$

для любого $n \geqslant 1$. Следовательно, $\prod_{1}^{2^{n}} G_{\tau^{n} \omega}^{\prime \prime \prime} \preceq \operatorname{Rist}_{G_{\omega}}(n)$ для любого $n \geqslant 1$. Заметим, что для любого $\omega \in \Omega_{\infty}$ подгруппа $G_{\omega}^{\prime \prime \prime}$ нетривиальна (поскольку $G_{\omega}$ неразрешима), а также имеет конечный индекс (поскольку $G_{\omega}$ минимально бесконечна). Таким образом, $\operatorname{Rist}_{G_{\omega}}(n)$ - подгруппа конечного индекса для любого $n \geqslant 1$.

Для непустого подмножества $\Lambda \subset \Omega$ обозначим через $U_{\Lambda}$ универсальную группу, соответствующую семейству $\left\{\left(G_{\omega}, S_{\omega}\right) \mid \omega \in \Lambda\right\}$. Для заданного $\Lambda \subset \Omega$ положим $T_{\Lambda}=\bigcup_{\omega \in \Lambda} T_{\omega}$; заметим, что $T_{\Lambda}$ - поддерево (не обязательно регулярное) дерева $T_{6}$. Отметим также, что $T_{\Lambda} U$-инвариантно (поскольку $U$-инвариантно любое $\left.T_{\omega}\right)$ и что сужение группы $U$ на $T_{\Lambda}$ дает универсальную группу $U_{\Lambda}$.

Предложение 5. Если $\Lambda \subset \Omega \backslash \Omega_{0}$, то группа $U_{\Lambda}$ с ее действием на $T_{\Lambda}$ является слабо почти ветвящейся группой.

Доказательство. Пусть $v \in T_{\Lambda}$, и пусть $v \in T_{\omega}$ для некоторого $\omega \in \Lambda$. Пусть $g$ - нетривиальный элемент жесткого стабилизатора $\operatorname{Rist}_{G_{\omega}}(v)$. Тогда в силу доказательства предложения 4 существует элемент $h \in \operatorname{Rist}_{U}(v)$, такой, что $\psi_{\omega}(h)=g$. Ограничение элемента $h$ на $T_{\Lambda}$ дает нетривиальный элемент из $\operatorname{Rist}_{U_{\Lambda}}(v)$. 


\section{§5. Универсальные группы промежуточного роста}

Цель этого параграфа - доказать существование подмножества $\Lambda \subset \Omega$ мощности $2^{\aleph_{0}}$, такого, что группа $U_{\Lambda}$ есть группа промежуточного роста. Этот факт был впервые установлен в работе [11], а здесь мы исправим неточность в приведенном там доказательстве.

Прежде всего кратко напомним основные понятия, связанные с ростом групп. Подробное изложение этой и близких тем имеется в работах [8], [18], [10].

Пусть $G$ - конечно порожденная группа, а $S$-конечное множество ее образующих. Длиной элемента $g \in G$ (относительно образующих $S$ ) называется число $\ell_{S}(g)=\min \left\{n \mid g=s_{1} \cdots s_{n}, s_{i} \in S^{ \pm}\right\}$. Функция роста группы $G$ (относительно $S$ ) определяется как $\gamma_{G, S}(n)=\# B(G, S, n)$, где $B(G, S, n)=\{g \in G \mid$ $\left.\ell_{S}(g) \leqslant n\right\}$ - шар радиуса $n$. Для двух возрастающих функций $f_{1}, f_{2}$ на множестве натуральных чисел будем писать $f_{1} \preceq f_{2}$, если существует постоянная $C>0$, такая, что $f_{1}(n) \leqslant f_{2}(C n)$ для всех $n$. Будем также писать $f_{1} \sim f_{2}$, если $f_{1} \preceq f_{2}$ и $f_{2} \preceq f_{1}$; это отношение является отношением эквивалентности. Можно заметить, что функции роста группы относительно различных конечных множеств образующих эквивалентны в смысле отношения , так что асимптотическое поведение функций роста группы является инвариантом самой группы.

Существуют три типа роста групп: если $\gamma_{G} \preceq n^{d}$ для некоторого $d \geqslant 0$, то говорят, что $G$ имеет полиномиальный рост, а если $\gamma_{G} \sim e^{n}$ - то экспоненциальный рост. Если не выполняется ни то, ни другое, то $G$ называется группой промежуточного роста.

Говоря о росте меченой группы $(G, S)$, мы обозначаем через $\gamma_{G}$ функцию роста группы $G$ относительно $S$.

Лемма 4. Пусть $F=\left\{\left(G_{i}, S_{i}\right) \mid i \in I\right\} \subset \mathscr{M}_{k}$ - непустое подмножсество. Обозначим через $\gamma_{F}$ функцию роста диагональной группъ $\left(U_{F}^{\mathrm{diag}}, S_{F}^{\mathrm{diag}}\right)$ из определения 3. Тогда справедливы следующие утверждения.

1. Для любого $i \in I \quad \gamma_{F}(n) \geqslant \gamma_{i}(n)$ для всех $n$.

2. Если I конечно, то $\gamma_{F}(n) \leqslant \prod_{i \in I} \gamma_{i}(n)$ для всех $n$.

Доказательство. В общем случае, если меченая группа $(H, K)$ является меченым образом меченой группы $(G, S)$, то $\gamma_{G}(n) \geqslant \gamma_{H}(n)$ для любого $n$. Поскольку все $\left(G_{i}, S_{i}\right)$ являются мечеными образами диагональной группы, мы получаем первое утверждение. Что касается второго утверждения, заметим, что $B\left(U_{F}^{\text {diag }}, S_{F}^{\text {diag }}, n\right) \subset \prod_{i \in I} B\left(G_{i}, S_{i}, n\right)$.

Для натурального числа $M$ через $\Omega_{M} \subset \Omega_{\infty}$ обозначим множество всех последовательностей, в которых каждое подслово длины $M$ содержит все символы $0,1,2$.

Теорема 6 [12, теорема 3.3]. Существуют постоянные $C$ и $\alpha<1$, зависящие только от $M$, такие, что если $\omega \in \Omega_{M}$, то

$$
\gamma_{G_{\omega}}(n) \leqslant C^{n^{\alpha}} \quad \text { для всех } n .
$$

Для заданных натуральных чисел $r_{1}, \ldots, r_{k}$ положим

$$
\Lambda_{r_{1}, \ldots, r_{k}}=\left\{(012)^{r_{1}} \eta_{1}(012)^{r_{2}} \eta_{2} \ldots(012)^{r_{k}} \eta_{k}(012)^{\infty} \mid \eta_{i} \in\{0,1,2\}\right\} \subset \Omega,
$$

где через $(012)^{\infty}$ обозначена периодическая последовательность 012012012 . . . 
Для последовательности $\mathbf{r}=\left\{r_{k}\right\}$ натуральных чисел положим

$$
\Lambda_{\mathbf{r}}=\left\{(012)^{r_{1}} \eta_{1}(012)^{r_{2}} \eta_{2} \ldots(012)^{r_{k}} \eta_{k} \ldots \mid \eta_{i} \in\{0,1,2\}\right\} \subset \Omega .
$$

Заметим, что и $\Lambda_{r_{1}, \ldots, r_{k}}$, и $\Lambda_{\mathbf{r}}$ суть подмножества в $\Omega_{4}$. Обозначим универсальные группы $U_{\Lambda_{r_{1}, \ldots, r_{k}}}$ и $U_{\Lambda_{\mathbf{r}}}$ соответственно через $U_{r_{1}, \ldots, r_{k}}$ и $U_{\mathbf{r}}$. Пусть $\gamma_{r_{1}, \ldots, r_{k}}$ и $\gamma_{\mathbf{r}}$ - функции роста этих групп (относительно канонических множеств образующих).

Лемма 5. Для заданных натуральных чисел $r_{1}, \ldots, r_{k}$ существует натуральное число $m$, такое, что

$$
\gamma_{r_{1}, \ldots, r_{k}, x}(m) \leqslant\left(1+\frac{1}{k}\right)^{m} \text { для любого } x \in \mathbb{N} .
$$

Доказательство. Так как $\Lambda_{r_{1}, \ldots, r_{k}, x} \subset \Omega_{4}$, то по теореме 6 существуют $C$ и $\alpha<1$ (не зависящие от $x$ ), такие, что

$$
\gamma_{\omega}(n) \leqslant C^{n^{\alpha}} \quad \text { при всех } n
$$

для всех $\omega \in \Lambda_{r_{1}, \ldots, r_{k}, x}$. Поэтому в силу леммы 4 (и того факта, что $\left|\Lambda_{r_{1}, \ldots, r_{k}, x}\right|=$ $\left.3^{k+1}\right)$ получаем

$$
\gamma_{r_{1}, \ldots, r_{k}, x}(n) \leqslant\left(C^{n^{\alpha}}\right)^{3^{k+1}}=D^{n^{\alpha}} \quad \text { для всех } n
$$

где $D=C^{3^{k+1}}$ не зависит от $x$. Значит, существует натуральное число $m$, такое, что

$$
\gamma_{r_{1}, \ldots, r_{k}, x}(m) \leqslant\left(1+\frac{1}{k}\right)^{m} \text { для любого } x \in \mathbb{N} \text {. }
$$

Лемма $6\left[11\right.$, лемма 3]. Пусть $\mathbf{r}=\left\{r_{k}\right\}$ - последовательность натуральнъх чисел. Если

$$
k+r_{1}+\cdots+r_{k} \geqslant \log _{2} 2 n
$$

для некоторого $k$, то $\gamma_{r_{1}, \ldots, r_{k}}(n)=\gamma_{\mathbf{r}}(n)$.

Теорема 7 [11, теорема 1]. Существует последовательность $\mathbf{r}=\left\{r_{k}\right\}$, maкая, что $U_{\mathbf{r}}$ - группа промежуточного роста.

Доказательство. Пусть $r_{1}=1$. По лемме 5 существует натуральное число $n_{1}$, такое, что

$$
\gamma_{r_{1}, x}\left(n_{1}\right) \leqslant\left(1+\frac{1}{1}\right)^{n_{1}} \text { для любого } x \text {. }
$$

Выберем $r_{2}$, такое, что $2+r_{1}+r_{2} \geqslant \log _{2} 2 n_{1}$. Снова по лемме 5 существует $n_{2}>n_{1}$, такое, что

$$
\gamma_{r_{1}, r_{2}, x}\left(n_{2}\right) \leqslant\left(1+\frac{1}{2}\right)^{n_{2}} \text { для любого } x .
$$

Предположим, что $r_{1}, \ldots, r_{k}$ уже выбраны. По лемме 5 существует $n_{k}>n_{k-1}$, такое, что

$$
\gamma_{r_{1}, \ldots, r_{k}, x}\left(n_{k}\right) \leqslant\left(1+\frac{1}{k}\right)^{n_{k}} \text { для любого } x \text {. }
$$

Выберем $r_{k+1}$, такое, что

$$
k+1+r_{1}+\cdots+r_{k+1} \geqslant \log _{2} 2 n_{k} .
$$


Рассуждая таким образом, мы построим последовательности $\mathbf{r}=\left\{r_{k}\right\}$ и $\left\{n_{k}\right\}$, для которых выполнены соотношения (3) и (4). Лемма 6 и соотношение (4) показывают, что

$$
\gamma_{r_{1}, \ldots, r_{k+1}}\left(n_{k}\right)=\gamma_{\mathbf{r}}\left(n_{k}\right)
$$

для всех $k$. Используя это соотношение и соотношение $(3)$, получаем

$$
\lim _{n \rightarrow \infty} \gamma_{\mathbf{r}}(n)^{1 / n}=\lim _{k \rightarrow \infty} \gamma_{\mathbf{r}}\left(n_{k}\right)^{1 / n_{k}}=\lim _{k \rightarrow \infty} \gamma_{r_{1}, \ldots, r_{k+1}}\left(n_{k}\right)^{1 / n_{k}} \leqslant \lim _{k \rightarrow \infty}\left(1+\frac{1}{k}\right)=1 .
$$

Следствие 2. Существует конечно порожденная группа промежуточного роста с $2^{\aleph_{0}}$ попарно не изоморфными гомоморфными образами.

Как уже упоминалось в начале параграфа, этот факт был установлен в [11] с небольшой неточностью. Наше доказательство в основном следует работе [11] с той единственной разницей, что нам требуется лемма 5.

\section{§6. Инвариантные случайные подгруппы универсальных групп}

Цель данного параграфа - показать, что существуют универсальные группы с большим числом инвариантных случайных подгрупп.

6.1. Предварительные сведения об инвариантных случайных подгруппах. Пусть $G$-счетная группа, и пусть $S(G)$ - пространство подгрупп группы $G$, снабженное топологией, базу которой образуют множества $\mathscr{O}_{A, B}=$ $\{N \leqslant G \mid A \subset N, B \cap N=\varnothing\}$, где $A, B$ - конечные подмножества в $G$. Пространство $S(G)$ можно отождествить с замкнутым подпространством в $\{0,1\}^{G}$, снабженным топологией, индуцированной топологией Тихонова. Группа $G$ действует на $S(G)$ сопряжениями, и это действие задает топологическую динамическую систему $(G, S(G))$. Нас интересует динамическая система вида $(G, S(G), \mu)$, где $\mu$-инвариантная относительно сопряжения вероятностная мера на $S(G)$.

Определение 7. Инвариантная относительно сопряжения борелевская вероятностная мера на $S(G)$ называется инвариантной случайной подгруппой (коротко ИC-подгруппой).

Пространство $S(G)$ представляет собой компактное метризуемое вполне несвязное пространство, состоящее (в соответствии с теоремой Кантора-Бендиксона $[16$, I.6]) из совершенного ядра $\kappa(G)$ и его счетного дополнения $S(G) \backslash \kappa(G)$. Совершенное ядро $\kappa(G)$ либо пусто, либо гомеоморфно канторову множеству, причем оно пусто тогда и только тогда, когда $S(G)$ счетно, т. е. у $G$ лишь счетное число подгрупп. Этот случай реализуется, например, для конечно порожденных почти нильпотентных групп, почти полициклических групп, некоторых метабелевых групп типа групп Баумслага-Солитера $B(1, n)$ и монстров Тарского [22].

Поскольку $\kappa(G)$ - инвариантное подмножество в $S(G)$ относительно действия группы $\operatorname{Aut}(G)$ и поскольку дополнение $S(G) \backslash \kappa(G)$ счетно, ясно, что непрерывная ИС-подгруппа обладает вероятностным законом $\mu$, сосредоточенным на $\kappa(G)$.

Для заданной подгруппы $L \leqslant G$ через $S(G, L) \subset S(G)$ обозначим множество подгрупп, содержащих $L$; ясно, что оно замкнуто. Заметим, что если $L-$ нормальная подгруппа в $G$, то множество $S(G, L)$ инвариантно относительно действия группы $G$ сопряжениями. 
Пусть $\varphi: G \rightarrow H-$ гомоморфизм. Он индуцирует два отображения

$$
\begin{aligned}
\bar{\varphi}: S(G) & \rightarrow S(H), \\
N & \mapsto \varphi(N),
\end{aligned}
$$

И

$$
\begin{aligned}
\widetilde{\varphi}: S(H) & \rightarrow S(G, \operatorname{Ker}(\varphi)), \\
K & \mapsto \varphi^{-1}(K) .
\end{aligned}
$$

Лемма 7. 1. Отображение $\bar{\varphi}$ борелевское.

2. Отображение $\widetilde{\varphi}$ непрерывно.

3. $\widetilde{\varphi}\left(K^{\varphi(g)}\right)=\widetilde{\varphi}(K)^{g}$ для всех $g \in G u K \leqslant H$.

4. $\widetilde{\varphi}^{-1}\left(C^{g}\right)=\widetilde{\varphi}^{-1}(C)^{\varphi(g)}$ для всех $g \in G u C \subset S(G, \operatorname{Ker}(\varphi))$.

5. Если гомоморфизм ч сюргективен, то $\tilde{\varphi}-$ гомеоморфизм.

Доказательство. 1. Мы утверждаем, что

$$
\bar{\varphi}^{-1}\left(\mathscr{O}_{A, B}\right)=\bigcap_{a \in A} \bigcup_{x \in \varphi^{-1}(a)} \bigcap_{y \in \varphi^{-1}(B)} \mathscr{O}_{\{x\},\{y\}},
$$

где $A$ и $B$-конечные подмножества в $H$.

Если $\varphi(N) \in \mathscr{O}_{A, B}$, то $A \subset \varphi(N)$ и $B \cap \varphi(N)=\varnothing$. Таким образом, для любого $a \in A$ существует $n_{a} \in N$, такое, что $\varphi\left(n_{a}\right)=a$. Кроме того, $y \notin N$ для любого $y \in \varphi^{-1}(B)$. Следовательно, $N$ принадлежит правой части.

Обратно, пусть $N \leqslant G$ принадлежит правой части. Это означает, что для любого $a \in A$ существует элемент $n_{a} \in \varphi^{-1}(a)$, такой, что $N \in \mathscr{O}_{\left\{n_{a}\right\},\{y\}}$ для всех $y \in \varphi^{-1}(B)$. Для любого $a \in A$ имеем $\varphi\left(n_{a}\right)=a$ и потому $A \subset \varphi(N)$. Кроме того, если $B \cap \varphi(N)$ непусто, то непусто и множество $N \cap \varphi^{-1}(B)$, что неверно. Поэтому $\varphi(N) \in \mathscr{O}_{A, B}$.

Заметим, что в общем случае отображение $\bar{\varphi}$ не является непрерывным. Например, последовательность подгрупп $(2 n+1) \mathbb{Z}, n \geqslant 1$, в $\mathbb{Z}$ сходится к тривиальной подгруппе, а их образы в $\mathbb{Z}_{2}$ сходятся ко всей группе

2. Мы утверждаем, что $\widetilde{\varphi}^{-1}\left(\mathscr{O}_{C, D}\right)=\mathscr{O}_{\varphi(C), \varphi(D)}$, где $C, D$ - конечные подмножества в $G$. Действительно, если $\tilde{\varphi}(K) \in \mathscr{O}_{C, D}$ для некоторой подгруппы $K \leqslant H$, то $C \subset \varphi^{-1}(K)$ и $D \cap \varphi^{-1}(K)=\varnothing$. Отсюда следует, что $\varphi(C) \subset K$ и $\varphi(D) \cap K=\varnothing$. Это, в свою очередь, показывает, что $K \in \mathscr{O}_{\varphi(C), \varphi(D)}$. Обратно, если $K \in \mathscr{O}_{\varphi(C), \varphi(D)}$ для некоторой подгруппы $K \leqslant H$, то $\varphi(C) \subset K$ и $D \cap \varphi(K)=\varnothing$. Отсюда следует, что $C \subset \varphi^{-1}(K)$ и $D \cap \varphi^{-1}(K)=\varnothing$, так что $\tilde{\varphi}(K)=\varphi^{-1}(K) \in \mathscr{O}_{C, D}$.

3. Это проверяется непосредственно.

4. Это следует из п. 2.

5. Если $\varphi$ сюръективен, то $\tilde{\varphi}$, очевидно, биективно. Поскольку множество $S(H)$ компактно, отсюда следует, что $\tilde{\varphi}$ - гомеоморфизм.

Следствие 3. Если $\mu$ есть ИС-подгруппа группы $H$, то мера $\nu=\widetilde{\varphi}_{*}(\mu)$ является ИС-подгруппой группы $G$ с носителем в множестве $\left\{\varphi^{-1}(K) \mid K \in\right.$ $\operatorname{supp}(\mu)\}$. Если, кроме того, $\mu$ непрерывна и эргодична относительно действия группы $H$ и отображение $\varphi$ сюрвективно, то мера $\nu$ непрерывна и эргодична относительно действия группы $G$. 
Доказательство. Первая часть является непосредственным следствием пп. 1 и 3 леммы 7. Заметим, что мера $\widetilde{\varphi}_{*}(\mu)$ определена на замкнутом подмножестве $S(G, \operatorname{Ker}(\varphi)) \subset S(G)$ и потому может рассматриваться как мера на $S(G)$ с носителем в $S(G, \operatorname{Ker}(\varphi)$. Предположим, что $\mu$ непрерывна и эргодична, а гомоморфизм $\varphi$ сюръективен. Так как $\tilde{\varphi}$ - гомеоморфизм, то мера $\nu$ непрерывна. Пусть $C \subset S(G, \operatorname{Ker}(\varphi))$ есть $G$-инвариантное подмножество. Для заданного $h \in H$ выберем элемент $g \in G$, такой, что $\varphi(g)=h$. Согласно п. 3 леммы $7, \widetilde{\varphi}^{-1}(C)^{h}=\widetilde{\varphi}^{-1}(C)^{\varphi(g)}=\widetilde{\varphi}^{-1}\left(C^{g}\right)=\widetilde{\varphi}^{-1}(C)$. Поэтому множество $\widetilde{\varphi}^{-1}(C) \quad H$-инвариантно, откуда следует, что $\nu(C)=\mu\left(\widetilde{\varphi}^{-1}(C)\right) \in\{0,1\}$.

Предложение 6. Пусть $X$-метрическое хаусдорфово топологическое пространство, и пусть $\mu$ - борелевская мера на $X$. Предположим также, что группа $G$ действует на борелевском пространстве $(X, \mu)$ сохраняющими меру преобразованиями. Тогда отображение St: $X \rightarrow S(G)$, задаваемое формулой $x \mapsto \mathrm{St}_{G}(x)$, борелевское. При этом мера $\nu=\mathrm{St}_{*}(\mu)$ является ИС-подгруппой с носителем в $\left\{\mathrm{St}_{G}(x) \mid x \in X\right\}$.

Доказательство. Заметим, что борелевская $\sigma$-алгебра на $S(G)$ порождается множествами вида $\mathscr{O}_{g}=\{N \leqslant G \mid g \in N\}$. Заметим также, что $\operatorname{St}^{-1}\left(\mathscr{O}_{g}\right)=$ $\operatorname{Fix}\left(\varphi_{g}\right)$, где отображение $\varphi_{g}: X \rightarrow X$ задано формулой $\varphi_{g}(x)=g . x$. Поэтому $\mathrm{St}^{-1}\left(\mathscr{O}_{g}\right)$ - борелевское множество (о сечениях борелевских отображений см., например, $\left[24\right.$, гл. 1]). Это показывает, что мера $\nu=\mathrm{St}_{*}(\mu)$ является борелевской мерой на $S(G)$ с носителем $\left\{\operatorname{St}_{G}(x) \mid x \in X\right\}$. Соотношение $\operatorname{St}_{G}(g \cdot x)=\operatorname{St}_{G}(x)^{g^{-1}}$ и $G$-инвариантность меры $\mu$ показывают, что $\nu$ инвариантна относительно сопряжения.

Известно (см. [1]), что любая ИС-подгруппа конечно порожденной группы возникает из сохраняющего меру действия на борелевском вероятностном пространстве $(X, \mu)$.

Граница $\partial T_{d} d$-регулярного корневого дерева $T_{d}$ определяется как множество всех бесконечных лучей, исходящих из корневой вершины. Граница $\partial T_{d}$ находится во взаимно однозначном соответствии с множеством бесконечных последовательностей над алфавитом $\{0,1, \ldots, d-1\}$ и потому гомеоморфна канторову множеству. Если $G$ - некоторая группа автоморфизмов корневого дерева $T_{d}$, то ее действие на $T_{d}$ продолжается до действия гомеоморфизмами на границе $\partial T_{d}$. Пусть $\mu$ - равномерная мера Бернулли на $\partial T_{d}$ (т. е. произведение счетного числа экземпляров равномерной меры на $\{0,1, \ldots, d-1\})$. Заметим, что $\mu$ непрерывна и инвариантна относительно действия группы $\operatorname{Aut}\left(T_{d}\right)$ и потому инвариантна относительно действия любой подгруппы $G \leqslant \operatorname{Aut}\left(T_{d}\right)$. О динамике таких действий известно следующее:

Предложение 7 [14]. Пусть $G$-счетная группа автоморфизмов регулярного корневого дерева $T_{d}$. Тогда следующие утверждения эквивалентны.

1. Группа $G$ действует транзитивно на уровнях дерева $T_{d}$.

2. Действие группы $G$ на $\partial T_{d}$ минимально (т.е. орбиты плотны).

3. Действие группы $G$ на $\partial T_{d}$ эргодично относительно равномерной меры Бернулли на $\partial T_{d}$.

4. Равномерная мера Бернулли является единственной $G$-инвариантной борелевской вероятностной мерой.

Действие слабо ветвящегося типа на $T$ порождает вполне несвободное действие на границе $\partial T$. 
Предложение 8 ([2], [14]). Пусть $G \leqslant \operatorname{Aut}(T)$ - слабо ветвящаяся группа. Тогда отображение $\mathrm{St}: \partial T \rightarrow S(G)$, задаваемое формулой $\xi \mapsto \operatorname{St}_{G}(\xi)$, инъективно.

Доказательство. Пусть $\xi, \eta \in \partial T$ - различные элементы границы дерева, и пусть $u, v$ - различные префиксы одинаковой длины $n$ элементов $\xi$ и $\eta$ соответственно. Бесконечные последовательности, начинающиеся с $u$ (соответственно с $v$ ), образуют окрестность элемента $\xi$ (соответственно $\eta$ ) в $\partial T$. Мы покажем, что стабилизатор $\mathrm{St}_{G}^{\circ}(\eta)$ этой окрестности элемента $\eta$ (который является подгруппой в $\left.\operatorname{St}_{G}(\eta)\right)$ не содержится в $\operatorname{St}_{G}(\xi)$.

Пусть $g \in \operatorname{Rist}_{G}(u)$ - нетривиальный элемент. Поскольку $v$ не содержится в поддереве $T_{u}$, любая бесконечная последовательность, начинающаяся с $v$, является неподвижной для $g$. Поскольку элемент $g$ нетривиален, он сдвигает некоторую вершину $u u_{1} \in T_{u}$, скажем, $g\left(u u_{1}\right)=u u_{2}$ для некоторых $u_{1} \neq u_{2}$ длины $m$. Пусть $u u^{\prime}$ - префикс элемента $\xi$ длины $n+m$.

Если $u^{\prime}=u$ или $u^{\prime}=u_{2}$, то $g\left(u u^{\prime}\right) \neq u u^{\prime}$ и поэтому $g \notin \operatorname{St}_{G}(\xi)$. Если и $u^{\prime} \neq u_{1}$, и $u^{\prime} \neq u_{2}$, то в силу уровневой транзитивности найдется элемент $h \in G$, такой, что $h\left(u u_{1}\right)=u u^{\prime}$. Тогда

$$
\left(h g h^{-1}\right)\left(u u^{\prime}\right)=(h g)\left(u u_{1}\right)=h\left(u u_{2}\right) \neq u u^{\prime},
$$

потому что $u_{1} \neq u_{2}$. Поэтому $h g h^{-1} \notin \operatorname{St}_{G}(\xi)$. Так как $h\left(u u_{1}\right)=u u_{2}$, то $h \in$ $\operatorname{St}_{G}(u)$ и потому $h g h^{-1} \in \operatorname{Rist}_{G}(u)$. Отсюда следует, что $h g h^{-1} \in \operatorname{St}_{G}^{\circ}(\eta)$.

Как объяснено во введении, отсюда немедленно получается непрерывная эргодическая ИС-подгруппа группы $G$. Детальное исследование этой и других связанных с нею мер на пространстве графов Шрейера группы «базилика» имеется, например, в [7].

Для действия групп Григорчука $G_{\omega}, \omega \in \Omega$, на границе $\partial T_{2}$ бинарного дерева мы получаем следующий результат:

Предложение 9. При $\omega \in \Omega$ действие группъ $G_{\omega}$ на $T_{2}$ транзитивно на уровнях, и поэтому действие группы $G_{\omega}$ на $\left(\partial T_{2}, \mu\right)$ эргодично. Таким образом, индуцированная ИС-подгруппа группь $G_{\omega}$ непреръвна и эргодична.

Доказательство. Согласно предложению 6 , действие группы $G_{\omega}$ на $\left(\partial T_{2}, \mu\right)$ индуцирует ИС-подгруппу группы $G_{\omega}$. Эта ИС-подгруппа непрерывна по предложению 8 и эргодична по предложению 7.

6.2. ИС-подгруппы универсальных групп. Для данных $\omega_{1}, \omega_{2} \in \Omega$ будем писать $\omega_{1} \sim \omega_{2}$, если существует перестановка $\sigma \in \operatorname{Sym}(\{0,1,2\})$, такая, что $\omega_{2}$ получается из $\omega_{1}$ ее применением к каждому символу в $\omega_{1}$. Напомним, что по теореме 1 , п. 7 , если $\omega_{1}, \omega_{2} \in \Omega_{\infty}$, то $G_{\omega_{1}} \cong G_{\omega_{2}}$ тогда и только тогда, когда $\omega_{1} \sim \omega_{2}$.

Для подмножества $\Lambda \subset \Omega$ через $|\Lambda|_{\sim}$ обозначим мощность множества классов эквивалентности отношения $\sim$ в $\Lambda$.

Предложение 10. Если $\Lambda \subset \Omega_{\infty}$, mо $U_{\Lambda}$ обладает как минимум $|\Lambda|_{\sim}$ различными непрерывными эргодическими ИС-подгруппами.

Доказательство. Зафиксируем $\Lambda \subset \Omega_{\infty}$. Пусть $\varphi_{\omega}: U_{\Lambda} \longrightarrow G_{\omega}$ - каноническая сюръекция, и пусть $N_{\omega}=\operatorname{Ker}\left(\varphi_{\omega}\right)$. Заметим, что если $\omega \nsim \eta$, то $N_{\eta} \not \leq N_{\omega}$, согласно п. 7 теоремы 1 с учетом того факта, что $G_{\eta}$ минимально бесконечна. 
Для $\omega \in \Omega$ и $\xi \in \partial T_{2}$ положим $W_{\omega, \xi}=\operatorname{St}_{G_{\omega}}(\xi)$. В силу предложения 9 каноническое действие группы $G_{\omega}$ на $\left(\partial T_{2}, \mu\right)$ индуцирует непрерывную эргодическую ИС-подгруппу $\mu_{\omega}$ на $G_{\omega}$, носитель которой содержится в $\left\{W_{\omega, \xi} \mid \xi \in \partial T_{2}\right\}$.

Пусть $\nu_{\omega}$ - индуцированная ИС-подгруппа в $U_{\Lambda}$, полученная, как описано в следствии 3 (т. е. $\left.\nu_{\omega}=\left(\tilde{\varphi}_{\omega}\right)_{*}\left(\mu_{\omega}\right)\right)$. Опять в силу следствия 3 мера $\nu_{\omega}$ непрерывна и эргодична. Положим $L_{\omega, \xi}=\varphi_{\omega}^{-1}\left(W_{\omega, \xi}\right)$ и заметим, что носитель ИС-подгруппы $\nu_{\omega}$ содержится в $Y_{\omega}=\left\{L_{\omega, \xi} \mid \xi \in \partial T_{2}\right\}$. Заметим также, что $L_{\omega, \xi}$ содержит $N_{\omega}$ для всех $\xi \in \partial T_{2}$.

Предположим, что $L_{\omega, \xi}=L_{\eta, \rho}$ для некоторых $\omega \nsim \eta \in \Lambda$ и $\xi, \rho \in \partial T_{2}$. Тогда $N_{\omega}, N_{\eta} \leqslant L_{\omega, \xi}$, и поэтому $L_{\omega, \xi}$ содержит подгруппу $N=N_{\omega} N_{\eta}$. Так как $N_{\eta} \not \leq N_{\omega}$, то $N_{\omega}$ - собственная подгруппа в $N$. Отсюда следует, что группа $U_{\Lambda} / N$ является собственной факторгруппой группы $U_{\Lambda} / N_{\omega} \cong G_{\omega}$. Поскольку $G_{\omega}$ - минимально бесконечная группа, $N$ и, следовательно, $L_{\omega, \xi}$ - подгруппы конечного индекса в $U_{\Lambda}$. Это, в свою очередь, показывает, что $\operatorname{St}_{G_{\omega}}(\xi)$ - подгруппа конечного индекса в $G_{\omega}$, и мы пришли к противоречию. Поэтому если $\omega \nsim \eta$, то носители мер $\nu_{\omega}$ и $\nu_{\eta}$ не пересекаются и, в частности, сами меры различны.

Комбинируя это с результатами $\S 5$, получаем основную теорему:

Основная теорема. Существует подмножество $\Lambda \subset \Omega$, такое, что coответствующая универсальная группа $U_{\Lambda}$ является группой промежуточного роста и обладает $2^{\aleph_{0}}$ различными непрерывными эргодическими инвариантными случайными подгруппами.

Благодарности. Авторы выражают благодарность А. М. Вершику и Я. Б. Воробцу за полезные обсуждения и рецензенту за тщательное чтение рукописи.

\section{ЛитеРАТУРА}

[1] M. Abért, Y. Glasner, B. Virág, Kesten's theorem for invariant random subgroups, Duke Math. J., 163:3 (2014), 465-488; http://arxiv.org/abs/1201.3399.

[2] L. Bartholdi, R. I. Grigorchuk, On parabolic subgroups and Hecke algebras of some fractal groups, Serdica Math. J., 28:1 (2002), 47-90.

[3] M. G. Benli, R. Grigorchuk, P. de la Harpe, Amenable groups without finitely presented amenable covers, Bull. Math. Sci., 3:1 (2013), 73-131.

[4] L. Bowen, R. Grigorchuk, R. Kravchenko, Invariant random subgroups of the lamplighter group, Israel J. Math., 207:2 (2015), 763-782; http://arxiv.org/abs/ 1206.6780 .

[5] L. Bowen, Invariant random subgroups of the free group, Groups, Geometry, Dynamics (в печати); http://arxiv.org/abs/1204.5939.

[6] C. Champetier, L'espace des groupes de type fini, Topology, 39:4 (2000), 657-680.

[7] D. D'Angeli, A. Donno, M. Matter, T. Nagnibeda, Schreier graphs of the Basilica group, J. Mod. Dyn., 4:1 (2010), 167-205.

[8] P. de la Harpe, Topics in Geometric Group Theory, Chicago Lectures in Math., University of Chicago Press, Chicago, IL, 2000.

[9] Р. И. Григорчук, В. В. Некрашевич, В. И. Сущанский, Автоматы, динамические системы и группы, в кн.: Динамические системы, автоматы и бесконечные группы, Сб. статей, Труды МИАН, т. 231, Наука, М., 2000, 134-214.

[10] R. I. Grigorchuk, Milnor's problem on the growth of groups and its consequences, in: Frontiers in Complex Dynamics, Princeton Math. Ser., vol. 51, Princeton Univ. Press, Princeton, NJ, 705-773; http://arxiv.org/pdf/1111.0512.pdf. 
[11] Р. И. Григорчук, Конструкиия р-групп промежуточного роста, обладающих континуумом факторгрупn, Алгебра и логика, 23:4 (1984), 383-394.

[12] Р. И. Григорчук, Степени роста конечно-порожденных групп и теория инвариантных средних, Изв. АН СССР, сер. матем., 48:5 (1984), 939-985.

[13] R. I. Grigorchuk, Just infinite branch groups, in: New horizons in pro- $p$ groups, Progr. Math., vol. 184, Birkhäuser, Boston, MA, 2000, 121-179.

[14] Р. И. Григорчук, Некоторые вопросы динамики групповых действий на корневых деревъях, в кн.: Современные проблемы математики, Сб. статей. K 75-летию Института, Труды МИАН, т. 273, МАИК, М., 2011, 72-191.

[15] R. Grigorchuk, Z. Šunić, Self-similarity and branching in group theory, in: Groups St. Andrews 2005, London Math. Soc. Lecture Note Series, vol. 339, Cambridge Univ. Press, Cambridge, 2007, 36-95.

[16] A. S. Kechris, Classical Descriptive Set Theory, Graduate Texts in Math., vol. 156, Springer-Verlag, New York, 1995.

[17] J. Lindenstrauss, G. Olsen, Y. Sternfeld, The Poulsen simplex, Ann. Inst. Fourier (Grenoble), 28:1 (1978), 91-114.

[18] A. Mann, How Groups Grow, London Math. Soc. Lecture Note Series, vol. 395, Cambridge University Press, Cambridge, 2012.

[19] J. Milnor, Problem 5603, in: Advanced Problems 5600-5609, Amer. Math. Monthly, 75:1 (1968), 685-686.

[20] R. Muchnik, Amenability of Universal 2-Grigorchuk group, http://arxiv.org/abs/ math/0505572.

[21] V. Nekrashevych, Self-Similar Groups, Mathematical Surveys and Monographs, vol. 117, Amer. Math. Soc., Providence, RI, 2005.

[22] А. Ю. Ольшанский, Бесконечная группа с подгруппами простых порядков, Изв. AH CCCP, сер. матем., 44:2 (1980), 309-321.

[23] D. V. Osin, Algebraic entropy of elementary amenable groups, Geom. Dedicata, 107 (2004), 133-151.

[24] K. R. Parthasaraty, Probability Measures on Metric Spaces (репринт оригинала 1967 г.), Amer. Math. Soc. Chelsia Publishing, Providence, RI, 2005.

[25] S. Sidki, Automorphisms of one-rooted trees: Growth, circuit structure and acyclicity, J. Math. Sci., 100:1 (2000), 1925-1943.

[26] А. М. Вершик, Несвободные действия счетных групп и их характеры, Зап. научн. сем. ПОМИ, 378 (2010), 5-16.

[27] A. M. Vershik, Totally nonfree actions and the infinite symmetric group, Mosc. Math. J., 12:1 (2012), 193-212, 216.

[28] Ya. Vorobets, Notes on the Schreier graphs of the Grigorchuk group, in: Dynamical Systems and Group Actions, Contemporary Math., vol. 567, Amer. Math. Soc., Providence, RI, 2012, 221-248.

Middle East Technical University, Ankara, Turkey

Поступила в редакцию e-mail: benli@metu.edu.tr 13 апреля 2015 г.

Texas A\&M University, College Station, TX, USA e-mail: grigorch@math.tamu.edu

Section de mathématiques, Université de Genève, Suisse e-mail: tatiana.smirnova-nagnibeda@unige.ch

Перевод на русский язык В. Е. Назайкинского 